\title{
Design and optimization of air core spiral resonators for magnetic coupling wireless power transfer on seawater
}

\author{
H. M. Santos, M. R. Pereira, L.M. Pessoa, H.M. Salgado
}

\begin{abstract}
This paper focuses on the design of high quality spiral resonators for maximising wireless power transfer efficiency between an AUV and an underwater docking station. By using 3D electromagnetic simulations and numerical analysis, the relevant parameters for quality factor computation are extracted. The impact of different variables on a spiral resonator's quality factor is assessed, allowing to conclude on the optimum design parameters to achieve optimum efficiency on the power transmission through magnetic coupling. This work will contribute to enable the development future AUV wireless charging systems, which will allow for an improvement of AUV's range and endurance while ensuring lower operational costs.
\end{abstract}

Index terms- Underwater wireless power transfer, magnetic coupling, spiral inductors, quality factor.

\section{INTRODUCTION}

Underwater vehicles, such as AUVs (Autonomous Underwater Vehicles), are capable of performing valuable missions such as search-and-rescue, surveillance, inspection, as well as monitoring water temperature and quality. These vehicles face range and endurance constraints, requiring periodic battery recharging. In near-surface operations, this process is often accomplished by a support vessel, which implies high operation costs when large scale oceanwide research is envisaged. Therefore, the development of underwater docking stations is extremely important to facilitate the recharging of AUVs while underwater. Currently available AUV recharging solutions are very complex, typically requiring "wet mate" connectors, which are prone to failure and require frequent maintenance and/or too complex docking mechanisms. As such, these solutions are not appropriate for scaling-up due to the high costs, and therefore their usage has been limited.

Despite the proven feasibility of wireless power transfer in air for various commercial and academic applications [1]-[3], few reports have addressed WPT in underwater media.

Although wireless power transfer methods have been reported for underwater operation, a thorough evaluation of coil quality factors' impact on efficiency has never been reported. The importance of such analysis lies in the fact that the figure of merit for such systems is given by FOM $=k Q$, where $k$ is the coupling coefficient and $Q$ the resonators' quality factor. Taking this into consideration, a method for maximizing the quality factor of spiral resonators is presented, which allows the performance improvement of an underwater WPT system. The method is based on a mathematical model obtained by fitting the resistance and inductance of the resonators extracted from 3D electromagnetic simulations. Additionally, resonator size constraints are also taken into account, considering its application inside a typical AUV. The paper is organized as follows: Section II presents the equivalent circuit model and Section III presents the proposed method. Results and discussion of the proposed modelling are then presented in Section IV while the paper's conclusion is given in Section V.

\section{Equivalent Circuit Model}

The equivalent circuit model of one shunt resonator can be given by the schematic in Fig. 1, as reported in [4]. In this

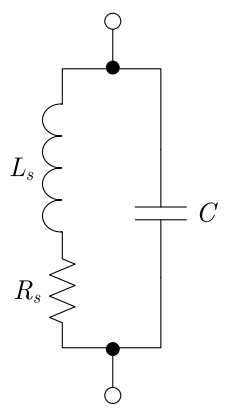

Fig. 1. Equivalent circuit model for a parallel resonator

equivalent circuit, $L_{s}$ represents the spiral's inductance and $R_{s}$ the sum of all series resistances, including DC, skin and proximity effects. The shunt capacitance can be adjusted to tune the resonator's frequency and is assumed to be ideal.

The quality factor for such resonator, when considering the capacitor ideal, is defined as [4]:

$$
Q=\frac{\omega_{0} L_{s}}{R_{s}} .
$$

However, in order to obtain the resonant angular frequency for this circuit, a series-parallel transformation of the RL is required, so that $\omega_{0}$ becomes $\omega_{0}=1 / \sqrt{L_{p} C}$, where $L_{p}$ is the equivalent parallel inductance. This transformation yields:

$$
Q=\frac{\sqrt{\frac{L_{s}-C R_{s}^{2}}{C}}}{R_{s}} .
$$

To assess the efficiency improvement with the employment of optimized resonators, two were brought to proximity for magnetic coupling to develop and power transfer to take place. The complete system's schematic is given in Fig. 2.

\section{PROPOSED METHOD}

Using 3D electromagnetic MoM simulation of the shunt resonator depicted in the model of Fig. 3, by resorting to HyperWorks FEKO, its input impedance can be obtained considering the equivalent circuit in Fig. 1. The resonator is enclosed within a PLA box that lies in seawater as the 


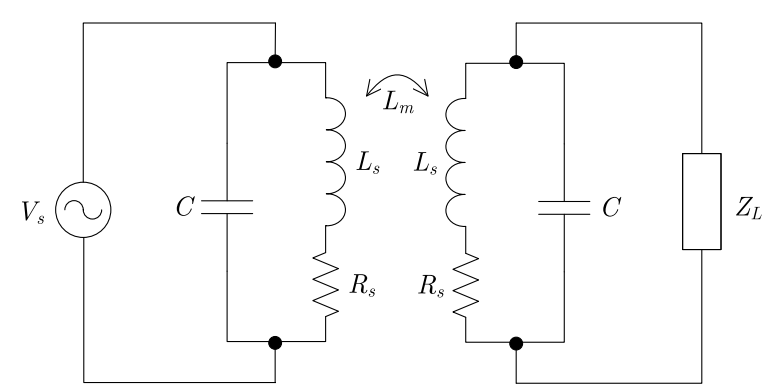

Fig. 2. Equivalent circuit model for a wireless power transfer system using spiral parallel resonators, in which $V_{s}$ represents the alternating power source and $Z_{L}$ the load impedance

free-space. For this particular case, the electrical properties of PLA were assumed to be $\epsilon_{r}=2.54$ and $\tan _{\delta}=7.4 \times 10^{-3}$, which are averages for the ranges of values reported in [5]. Seawater properties are exploited in [6], where an average value of $\sigma=4 \mathrm{~S} / \mathrm{m}$ is considered for conductivity and relative permittivity is chosen to be $\epsilon_{r}=81$.

Multiple simulations were carried out by sweeping the tuning capacitance for different numbers of wire turns, allowing for two dimensional evaluation of the quality factor, namely as a function of the resonant frequency and the number of turns. The inner and outer diameters were held constant, due to space limitation in the application specific AUVs. By solving

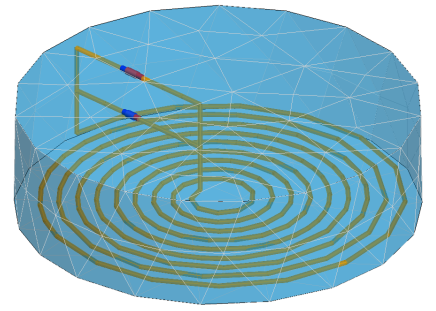

Fig. 3. FEKO model for 3D electromagnetic simulation

the subjacent algebra for the resonator's input impedance, considering the schematic depicted in Fig. 1, we obtain:

$$
\operatorname{Re}\left(Z_{i n}\right)=\frac{R_{s}}{R_{s}^{2} C^{2} \omega^{2}+\left(\omega^{2} L_{s} C-1\right)^{2}}
$$

and

$$
\operatorname{Im}\left(Z_{i n}\right)=-\frac{\omega\left(C R_{s}^{2}+\left(\omega^{2} L_{s} C-1\right) L_{s}\right)}{R_{s}^{2} C^{2} \omega^{2}+\left(\omega^{2} L_{s} C-1\right)^{2}} .
$$

From simulation results, both real and imaginary parts of the resonator's input impedance can then be extracted. By analysing the simulation's outcome, the zero crossing frequency was obtained for the imaginary part. Choosing the closest data point to the zero crossing, one knows the imaginary and real parts of the input impedance, as well as the corresponding angular frequency $\omega$. Solving a system of equations composed by (3) and (4) for the two unknowns, $R_{s}$ and $L_{s}$, results in the following resistance and inductance:

$$
\begin{aligned}
& R_{s}= \\
& \frac{\operatorname{Re}\left(Z_{i n}\right)}{\left(\operatorname{Re}\left(Z_{i n}\right)^{2}+\operatorname{Im}\left(Z_{i n}\right)^{2}\right) \omega^{2} C^{2}+2 \operatorname{Im}\left(Z_{i n}\right) \omega C+1}
\end{aligned}
$$

and

$$
\begin{aligned}
& L_{s}= \\
& \frac{\left(\operatorname{Re}\left(Z_{i n}\right)^{2}+\operatorname{Im}\left(Z_{i n}\right)^{2}\right) \omega C+\operatorname{Im}\left(Z_{i n}\right)}{\omega\left[\left(\operatorname{Re}\left(Z_{i n}\right)^{2}+\operatorname{Im}\left(Z_{i n}\right)^{2}\right) \omega^{2} C^{2}+2 \operatorname{Im}\left(Z_{i n}\right) \omega C+1\right]} .
\end{aligned}
$$

Considering that all the variables present in equations (5) and (6) are known, the tuning capacitance, $C$, was swept from $1 \mathrm{nF}$ to $625 \mathrm{nF}$ and the number of turns, $N$, varied between 5 and 15 . The wire diameter was chosen to be $2 \mathrm{~mm}$. The resonators' inner and outer diameters were considered to be $d_{\text {inner }}=2 \mathrm{~cm}$ and $D_{\text {outer }}=16 \mathrm{~cm}$, respectively. These values are determined by the AUV's size restrictions, for which this system is targeted.

\section{RESUlts}

By applying the method presented in section III, it was possible to obtain different values of resistances and inductances, for multiple resonant frequencies and number of turns.

In Fig. 4, AC resistance, $R_{s}$, for different number of turns as a function of the resonant frequency is depicted. A quadratic model that fits the data points for each number of turns is also presented. By extrapolating from the quadratic fitting for each number of turns, it was observed that the DC series resistance is given by the well known expression:

$$
R_{D C}=\rho \frac{L}{A},
$$

where $A$ corresponds to the wire's section area and $L$ corresponds to the total length wound in a spiral shape. Due to the frequency increment, as described in [7], [8], the resistance increases in a quadratic proportion due to current-crowding effects, which validates our fitting. Also, by increasing the number of turns, we verified that resistance also increases for a constant frequency. This increment is justified with increasing eddy current losses because of the diminishing space between turns. The spiral inductance for different number of turns was also seen to present a quadratic frequency dependence as it can be observed in Fig. 5. This inductance decrease, as well as the resistance increase depicted in Fig. 4, is also explained by non-uniformities in the current that contributes to the magnetic field. In fact, a more pronounced current distribution starts to develop with increasing frequency, which results in a nonhomogeneous magnetic field and so a frequency dependent inductance. To support the proposed model, it was also verified that, for DC, the obtained inductance values are very close to the theoretical prediction for each number of turns. A comparison between the analytical model and the simulation results for the input impedance was made, which resulted in a maximum relative error of $e_{\max }=8.9 \%$ and an RMS of $e_{R M S}=6.26 \%$. For this calculations, the nominal value was assumed to be the one provided from the simulation results. 


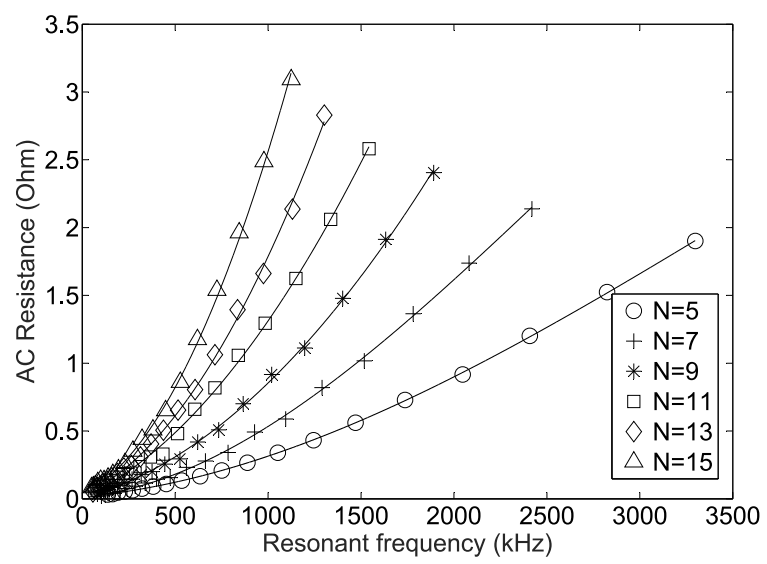

Fig. 4. Data and fitted quadratic models obtained from simulation for AC resistance as a function of resonant frequency

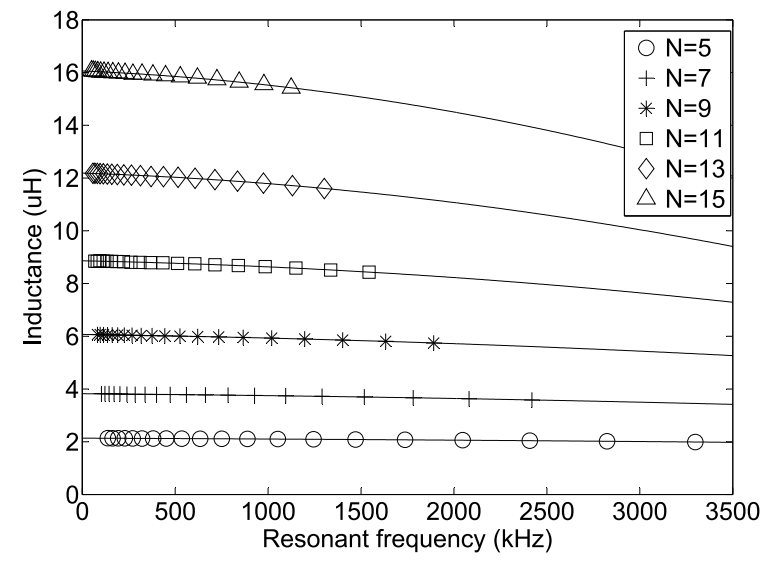

Fig. 5. Data and fitted quadratic models obtained from simulation for inductance as a function of resonant frequency

The quality factors present in Fig. 6 were obtained by employing the fitted curves shown in Figs. 4 and 5, in equation (2). By inspection of the result present in Fig. 6, we concluded that for each $N$, a maximum for the quality factor occurs. With increasing resonant frequency, the resonator's $Q$ increases as expected by equation (2). However, at a certain point, the series resistance also starts to increase until it lowers the quality factor, explaining the peaks observed. Noticeably, for higher frequencies, less turns lead to an increase in the $Q$ factor, as eddy current losses are less significant due to higher spacing between turns. Nonetheless, current-crowding (skineffect) still exists, which continuously decreases the quality factor as frequency rises.

The impact of optimising the quality factor was also evaluated on the wireless power transfer efficiency, as shown in Fig. 7. It can be concluded that increasing the $\mathrm{Q}$ factor leads to higher transfer efficiency (up to $95 \%$ ) in close distance, while lower Q factor is suitable for keeping high efficiencies for larger distances.

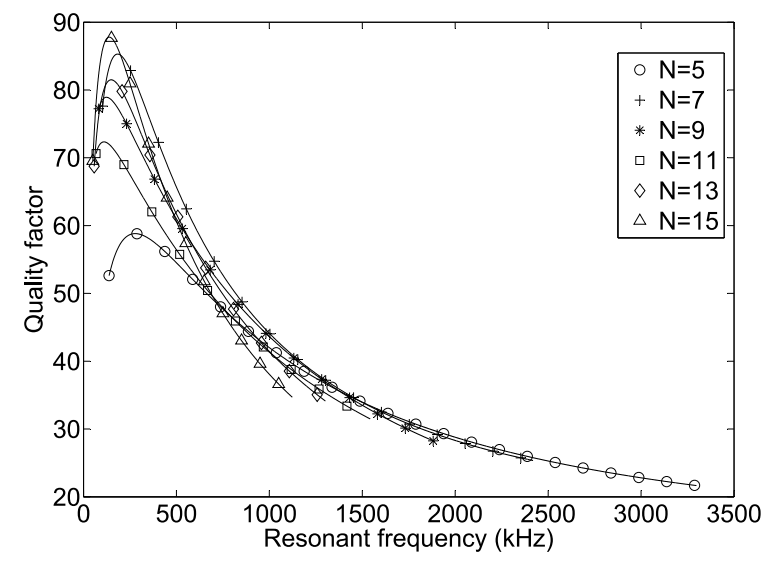

Fig. 6. Quality factor as a function of resonant frequency for different numbers of turns

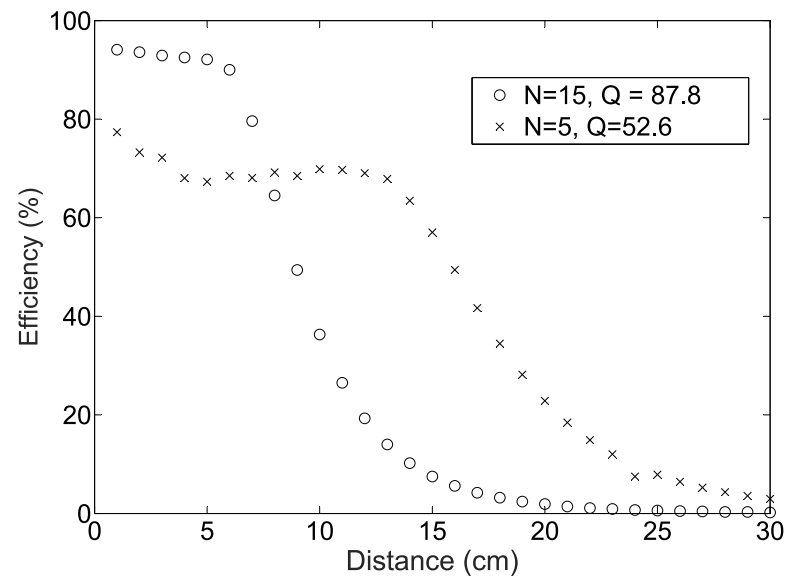

Fig. 7. Efficiencies in terms of distance between resonators

\section{CONClusion}

In this paper a new method to design high quality spiral resonators for maximising wireless power transfer in an underwater scenario is proposed. A validation has been accomplished for the proposed model by comparing its results with simulation outputs. The presented numerical model is suitable to determine the global maximum of the quality factor as a function of operating frequency and number of turns. A wireless power transfer efficiency up to $95 \%$ was obtained when considering the optimised spiral resonators as well as an unloaded quality factor of $Q=87.8$.

\section{ACKNOWLEDGEMENTS}

This work has been developed as part of project ENDURE - Enabling Long-Term Deployments of Underwater Robotic Platforms in Remote Oceanic Locations. ENDURE (PT02 Aviso4 0015) benefits from a 218157 grant from Iceland, Liechtenstein and Norway through the EEA Grants. The aim of the programme PT02 is to achieve a good environmental status, in accordance with the Marine Strategy Framework 
Directive and one of the expected outputs consists in the strengthening of the capacity of mobile remote monitoring ocean through the introduction of innovative supporting platforms. This work was also carried out with the support of the TEC4SEA research infrastructure (www.tec4sea.com), and the support of COST action IC1301 - WIPE.

\section{REFERENCES}

[1] M. Kesler, "Highly resonant wireless power transfer: Safe, efficient, and over distance," in WiTricity Corporation, 2013.

[2] S.-M. Kim, I.-K. Cho, J.-I. Moon, J.-H. Yoon, W.-J. Byun, and J. ick Choi, "1.9MHz wireless power transmission system using coupled magnetic resonance," in Microwave Radar and Wireless Communications (MIKON), 2012 19th International Conference on, vol. 2, May 2012, pp. 519-522.

[3] Z. Bin and H. Xiao-hong, "Modeling and analysis of wireless power transmission system via strongly coupled magnetic resonances," in Mechatronics and Control (ICMC), 2014 International Conference on, July 2014, pp. 70-75.

[4] A. Sample, D. Meyer, and J. Smith, "Analysis, experimental results, and range adaptation of magnetically coupled resonators for wireless power transfer," Industrial Electronics, IEEE Transactions on, vol. 58, no. 2, pp. 544-554, Feb 2011.

[5] K. Shinyama and S. Fujita, "Study on the electrical properties of a biodegradable plastic," in Properties and Applications of Dielectric Materials, 2003. Proceedings of the 7th International Conference on, vol. 2, June 2003, pp. 707-710 vol.2.

[6] E. A. Karagianni, "Electromagnetic waves under sea: bow-tie antennas design for wi-fi underwater communications," in Progress In Electromagnetics Research M, vol. 41, 2015, pp. 189-198.

[7] H.-M. Hsu, "Effective series-resistance model of spiral inductors," Microwave and Optical Technology Letters, vol. 46, no. 2, pp. 107-109, July 2005.

[8] W. Kuhn, "Approximate analytical modeling of current crowding effects in multi-turn spiral inductors," in Radio Frequency Integrated Circuits (RFIC) Symposium, 2000. Digest of Papers. 2000 IEEE, June 2000, pp. 271-274. 